\title{
Archives
}

\section{CHEYROU, C. (2015). Les Ursulines de Québec. Espaces et mémoires. Québec, Québec : Fides, 211 pages}

\section{Laure Amélie Guitard}

Volume 47, numéro 1, 2017

URI : https://id.erudit.org/iderudit/1041832ar

DOI : https://doi.org/10.7202/1041832ar

Aller au sommaire du numéro

Éditeur(s)

Association des archivistes du Québec (AAQ)

ISSN

0044-9423 (imprimé)

2369-9256 (numérique)

Découvrir la revue

Citer ce compte rendu

Guitard, L. A. (2017). Compte rendu de [CHEYROU, C. (2015). Les Ursulines de Québec. Espaces et mémoires. Québec, Québec : Fides, 211 pages]. Archives,

47(1), 193-198. https://doi.org/10.7202/1041832ar

Ce document est protégé par la loi sur le droit d'auteur. L’utilisation des services d’Érudit (y compris la reproduction) est assujettie à sa politique d'utilisation que vous pouvez consulter en ligne.

https://apropos.erudit.org/fr/usagers/politique-dutilisation/
Cet article est diffusé et préservé par Érudit.

Érudit est un consortium interuniversitaire sans but lucratif composé de l’Université de Montréal, l'Université Laval et l'Université du Québec à Montréal. Il a pour mission la promotion et la valorisation de la recherche. https://www.erudit.org/fr/ 


\section{COMPTE RENDU}

\section{CHEYROU, C. (2015). Les Ursulines de Québec. Espaces et mémoires. Québec, Québec: Fides, 211 pages.}

\section{LAURE AMÉLIE GUITARD}

Candidate au doctorat en sciences de l'information, École de bibliothéconomie et des sciences de l'information, Université de Montréal

Christine Cheyrou a réalisé Les Ursulines de Québec en collaboration avec I'historien Julien Mercure-Gauvin, les Ursulines, le Musée des Ursulines de Québec et les Archives du Monastère des Ursulines de Québec. Membre du Conseil du patrimoine culturel du Québec, elle est directrice et conservatrice du Musée des Ursulines de Québec depuis 1993 et auparavant archiviste de référence aux Archives du Monastère des Ursulines de Québec. Paru aux éditions Fides, cet ouvrage porte sur le patrimoine des Ursulines de Québec,

... appréhendé sous l'angle de la mémoire pour montrer comment ce patrimoine monastique, qu'il soit matériel ou intangible, s'est construit, se définit et se pérennise dans une structure et une construction mémorielles (p. 9).

C'est à partir du phénomène de la mémoire observé au sein de la communauté encore vivante que les bases de sa conservation et de sa 
transmission sont posées (p. 9). Cet ouvrage s'adresse au grand public et a pour

\begin{abstract}
... objectif principal de rendre accessibles un contexte historique et des mécanismes de mémoire qui nous aident en tant que société à comprendre, à percevoir, à apprécier et même à aimer ce patrimoine singulier, longtemps tenu secret, dont nous sommes aussi les héritiers (p. 16).
\end{abstract}

Monique Pelletier, OSU1', présidente du conseil de gestion, résume parfaitement dans la préface l'essence de cet ouvrage: « en parcourant les pages qui suivent, vous entrez en contact avec l'âme de la communauté ursuline» (p. 3).

Au premier abord, le lecteur est séduit par le côté esthétique de I'ouvrage. Il est illustré de reproductions de documents d'archives de plusieurs types (textuels, iconographiques, cartes et plans), de photographies d'objets, de peintures du Musée des Ursulines ou de bâtiments du complexe monastique. La structure tripartite permet de traiter progressivement le concept de mémoire à partir de l'exemple du patrimoine matériel et immatériel des Ursulines de Québec: la constitution de la mémoire, les formes de la mémoire et la transmission de la mémoire. Trois encadrés sur des notions clés ponctuent la lecture: les annales, histoire et mémoire et le patrimoine immatériel. Rédigés par Julien Mercure-Gauvin, historien, ils offrent un recul qui situe la réalité des ursulines dans la réalité des communautés religieuses du Québec.

La mémoire des Ursulines de Québec prend ses racines dans la fondation de la communauté en Nouvelle-France au XVII siècle. Le récit de l'arrivée des fondatrices, appelé Vieux Récit, a survécu aux incendies et les affres du temps grâce à sa transmission de mémoire en mémoire. Le patrimoine de la communauté est formé de la Règle de Saint-Augustin commune à plusieurs communautés à laquelle s'ajoute l' « héritage spirituel et historique des devancières par leur mémoire sans cesse remémorée » (p. 42). C'est ce dernier élément qui permet de rendre unique la mémoire de la communauté. Le récit authentique perdu dans les flammes n'est pas identique au récit reconstitué de mémoire - la mémoire des derniers témoins vivants. Mais ce patrimoine est tout aussi précieux, car il fonde l'identité de la communauté. Les documents d'archives ne sont pas les seuls vecteurs de la mémoire des Ursulines. Le patrimoine est envisagé 
dans son ensemble: biens matériels et immatériels conservés et décrits pour rendre compte de l'histoire de l'institution.

Bien que l'ouvrage comprenne une préface, un avant-propos et une introduction, il est difficile pour le lecteur d'entrer dans le texte. L'introduction le prévient justement sur ce point: les codes et le vocabulaire sont difficiles à pénétrer (p. 16). Certaines reproductions de plan sont malheureusement illisibles parce que trop petites. Certains documents iconographiques ne sont pas toujours bien intégrés au texte. Ils manquent alors de contexte. Par exemple, la photographie du grenier aux coffres (p. 35) reste anodine pour qui ne sait pas que chacun des coffres appartient à une religieuse, seule propriété individuelle dans la communauté, reliquat de sa mémoire personnelle au sein de l'institution communautaire. Ceci est expliqué au lecteur beaucoup plus loin (p. 105). Des définitions telles que «annales» (p. 17-18) sont illustrées en tant que vocabulaire de la religion et avec des précisions sur l'emploi propre aux Ursulines de Québec. II manquerait cependant certains repères historiques au novice en histoire du Québec: la Compagnie des Cent Associés, par exemple. Certaines expressions telles que "des «mains» de papier» (p. 134) ne sont pas expliquées. Ce qui laisse le lecteur sur sa faim. De même, certains événements manquent de contexte. Par exemple, le procès de la Corriveau est évoqué dans un témoignage, mais pas plus d'explications ni de contexte ne sont donnés au lecteur qui ne serait pas familier avec cette figure du folklore québécois.

Plusieurs typographies permettent de distinguer plusieurs types de discours qui s'entremêlent pour offrir un paysage diversifié des sources de savoir sur le patrimoine des ursulines: I'auteure, des historiens, des universitaires en religion et en architecture, mais aussi des religieuses dont les entrevues pour des travaux antérieurs ont été retranscrites partiellement. La dernière section avant la conclusion a été rédigée à partir des témoignages de deux religieuses. De la forme de discours indirect libre, elle donne des indications sur la manière dont les religieuses vivaient de l'intérieur l'appel à l'enseignement. Rédigée au «nous», elle parachève l'immersion dans la vie ursuline. II est dommage cependant que les témoins interrogés ne soient pas décrits; un paragraphe est accordé en fin d'ouvrage à la description des projets dans le cadre desquels ont eu lieu les entrevues, mais on ne sait pas qui a été interrogé (par ex., leur statut dans la communauté). Cette grande diversité de styles porte parfois à confusion ou du moins déstabilise le lecteur, allant d'un 
style oral retranscrit au discours scientifique en passant par des extraits de correspondance et d'annales du XVII siècle. Mais tous ces discours convergent pour témoigner de l'œuvre de la communauté religieuse, dans un seul lieu, à travers le temps et les personnes.

L'auteure a recours à des documents d'autres communautés - par exemple, les Relations des Jésuites - et offre ainsi une histoire de la communauté dans un contexte plus large, un contexte qui tient compte des autres communautés et de la réalité politique et économique passée.

De l'émotion se dégage tant du récit de l'histoire de la communauté que des descriptions qui rendent les objets presque palpables. Les témoignages oraux et le partage de connaissances participent à la création d'une atmosphère particulière: le lecteur entre dans l'intimité de la communauté par l'émotion qui émane des documents. En effet, l'aspect intime de la vie du créateur d'archives a été perçu comme un des facteurs déclencheurs de l'émotion (Klein, Dufour et Mas, 2014). La dimension émotive des archives (Lemay et Boucher, 2010; Lemay et Klein, 2012) joue ainsi un rôle dans l'approche mémorielle de la sauvegarde du patrimoine immatériel.

L'ouvrage est le fruit de la longue expérience de Christine Cheyrou auprès des Ursulines de Québec. Il est une mine d'or pour qui s'intéresse à cette communauté et à son patrimoine. En outre, il est un exemple de mise en valeur du patrimoine d'une communauté religieuse que d'autres communautés pourraient suivre. Finalement, en considérant à la fois les biens matériels et immatériels de la communauté, il est un exemple de mise en valeur du patrimoine archivistique situé dans l'ensemble des connaissances patrimoniales et mémorielles d'une institution.

Ce travail d'exploitation des archives associées à l'architecture et aux témoignages s'inscrit dans une dynamique de valorisation des archives où les archivistes font preuve de créativité. Ainsi, selon Nicolas Bednarz et Céline Widmer, le rôle de l'archiviste « doit s'étendre à la transmission d'une vision personnelle du patrimoine» (Bednarz et Widmer, 2015, p. 118). Erika Nimis voit dans I'utilisation artistique des archives une reconstitution de l'histoire qu'on a oubliée. La nouvelle exploitation des archives permet une actualisation du message qu'elles véhiculent, de leur signification (Lemay et Klein, 2015, p. 9). L'auteure rejoint la préoccupation des Ursulines de transmettre une histoire de leur communauté, même si 
celle-ci n'est pas absolument véridique. La transmission de la mémoire est considérée non pas comme la transmission de l'histoire, mais comme la transmission des mémoires, de l'histoire commune d'un groupe que chaque personne raconte à sa manière.

La question qui se pose est donc de savoir comment cette mémoire collective toujours présente sera pérennisée après la disparition, une à une, des mémoires individuelles de la communauté, bref, comment garder vivante la mémoire de la vie? (p. 206)

II n'est pas étonnant qu'une communauté dont la mission est l'enseignement soit aussi attachée à la mémoire et à la transmission de cette mémoire. La mémoire orale, écrite, artéfactuelle et l'ensemble des connaissances des Ursulines de Québec sont «un trésor documentaire d'autant plus précieux qu'il est menacé par la disparition de ses interprètes » (p. 167-168). Cet ouvrage participe à la médiation historique (Clouet, 2012) de la communauté en explicitant le sens attribué aux documents d'archives, aux objets, aux personnes, bref, aux vies qui la formaient et la forment encore.

LAURe Amélie Guitard

\section{NOTES}

1. «OSU » est une abréviation courante pour «ordre de Sainte-Ursule ».

\section{BIBLIOGRAPHIE}

BEDNARZ, N. et WIDMER, C. (2015). Archives au pluriel: le Montréal de 1914-1918. L'expérience d'une création collaborative et multidisciplinaire. Dans Y. Lemay et A. Klein (dir.), Archives et création: nouvelles perspectives sur l'archivistique. Cahier 2 (p. 112-142). Montréal, Québec: Université de Montréal, École de bibliothéconomie et des sciences de l'information (EBSI). 
CLOUET, C. (2012). Quelle(s) médiation(s) pour l'histoire? Bulletin des bibliothèques de France, 2 . Repéré à http://bbf.enssib.fr/consulter/ bbf-2012-02-0085-009

KLEIN, A., DUFOUR, C. et MAS, S. (2014). Émouvantes les archives? Le point de vue des archivistes. La Gazette des archives 233, 75-90.

LEMAY, Y. et BOUCHER, M.-P. (2010-2011). L'émotion ou la face cachée de l'archive ( $6^{\mathrm{e}}$ symposium du Groupe interdisciplinaire de recherche en archivistique (GIRA)). Archives 42(2), 39-52.

LEMAY, Y. et KLEIN, A. (2012). Archives et émotions. Documentation et bibliothèques 58(1), 5-16.

LEMAY, Y et KLEIN, A. (dir.) (2015). Archives et création: nouvelles perspectives sur l'archivistique. Cahier 2. Montréal, Québec: Université de Montréal, École de bibliothéconomie et des sciences de l'information (EBSI). Repéré à http://hdl.handle.net/1866/12267 